\title{
Suppression of invasive characteristics by antisense introduction of overexpressed HOX genes in ovarian cancer cells
}

\author{
TSUYOSHI YAMASHITA, SEISHIRO TAZAWA, ZHAO YAWEI, HIDETO KATAYAMA, \\ YASUHITO KATO, KUNIHIKO NISHIWAKI, YUKO YOKOHAMA and MUTSUO ISHIKAWA \\ Department of Obstetrics and Gynecology, Asahikawa Medical College, \\ Midorigaoka, Higashi 2-1-1-1, Asahikawa 078-8510, Japan
}

Received October 24, 2005; Accepted December 12, 2005

\begin{abstract}
HOX genes encode transcription factors that function to establish basic body pattern during embryogenesis and maintain the function of specific organs in the adult. Recent studies have demonstrated that HOX genes are also involved in oncogenesis in a range of malignancies. To elucidate whether HOX genes contribute to ovarian carcinogenesis, we created an expression profile of HOX genes using ovarian derived materials from surgical samples and epithelial ovarian cancer cells derived from five different cell lines. Real-time quantitative RT-PCR assay indicated overexpression of $14 \mathrm{HOX}$ genes in clusters A and B but only 2 genes in clusters $\mathrm{C}$ and $\mathrm{D}$. Of the $16 \mathrm{HOX}$ genes, overexpression of paralogs of HOX3, HOX 4 and $\mathrm{HOX} 7$ is seen in cluster A and B, and of HOX13 in all paralogs. In addition, HOXB7, HOXA13 and HOXB13 showed high levels of overexpression in cancer cells and tissues whereas no or little expression was observed in normal controls. To examine whether overexpressed HOX genes regulate invasion of ovarian cancer cells directly, we introduced an antisense DNA fragment of overexpressed HOXB7 and HOXB13, and HOXC5 that did not show overexpression into SKOV3 cells by electroporation. Antisense introduction followed by chemoinvasion assay using matrigel chamber demonstrated that SKOV3 cells introduced an antisense of each HOXB7 and HOXB13 showed $85 \%$ and $50 \%$ reduction of invasion ability compared to the parental SKOV3 cells, respectively. In contrast, antisense of HOXC5 introduced cells showed no significant difference of the invasion ability. These results suggest an important role of overexpressed HOX genes, especially for invasive characteristics of ovarian cancer cells.
\end{abstract}

Correspondence to: Dr Tsuyoshi Yamashita, Department of Obstetrics and Gynecology, Asahikawa Medical College, Midorigaoka, Higashi 2-1-1-1, Asahikawa 078-8510, Japan E-mail: tyamashi@asahikawa-med.ac.jp

Key words: HOX, ovarian cancer, invasion, antisense, electroporation

\section{Introduction}

Epithelial ovarian cancer is a significant female disease with a high rate of mortality, mainly because most patients are diagnosed at advanced clinical stages due to their delay in visiting a hospital and the easy diffusion of cancer cells into pelvic and abdominal cavities (1). Thus, elucidating the mechanisms of invasion and metastasis in ovarian cancer is essential for understanding the nature of ovarian cancer. In order to invade or metastasize, the cancer cells must move from the ovarian surface lesion, inside or outside of the ovary as a primary site, to secondary metastatic sites. This involves detachment from neighboring cells, transfer of the cells after intravasation, and then proliferation after extravasation. This complicated functional alteration of the cells requires changes in the characteristics of the cells, such as the phenomenon seen in epithelial mesenchymal transition (EMT) regulated by several molecules such as TGF, Ras, Wnt and Snail (2-10). In early embryogenesis, these molecules are also utilized to form the basic embryonic body pattern via EMT. HOX genes, encoding homeodomain as DNA-binding domain and consisting of 39 members in four different clusters, are one type of such regulatory transcription molecules that express in the anterior-posterior axis, limbs, and genital buds during mammalian embryogenesis. Published studies suggest that HOX genes provide intercellular information for the determination of identity, lineage and fate of the cells $(11,12)$.

Interestingly, recent reports have provided evidence that the abnormal expression of certain HOX genes is observed in a variety of malignancies such as hematologic, breast, colon, prostate, bladder, lung, and thyroid cancer (13-22). In the gynecologic field, there are several reports describing that abnormal expression of particular HOX genes seems to be involved in cervical, ovarian and endometrial neoplasms (23-26) including a recent report by Zhao et al (27). In the context of embryonic development, the expression of invasive characteristics of cancer cells might be a similar phenomenon, analogous to the movement of normal embryonic cells mediated by expression of HOX genes. This leads to a possibility that overexpression of certain HOX genes in ovarian cancer cells could accelerate invasion of the cancer cells.

In this study, we first created an expression profile of HOX genes in ovarian-derived samples to investigate whether 
Table I. Primer sequences of $36 \mathrm{HOX}$ genes for real-time PCR.

\begin{tabular}{|c|c|c|c|c|c|}
\hline Gene & & & Gene & & \\
\hline \multirow[t]{2}{*}{ A1 } & Sense: & AGTTGGAGAGTACGGCTACCTG & $\mathrm{C} 4$ & Sense: & ACCGTCGCATGAAATGGAAG \\
\hline & Antisense: & : TGCAGGGATGCAGCGATCTCCAC & & Antisense: & CTGGGAGTGGTCTTCAGAAGTACC \\
\hline \multirow[t]{2}{*}{$\mathrm{A} 2$} & Sense: & CTCTTTGCAGGACTTTAGCGTT & $\mathrm{C} 5$ & Sense: & AGATTTACCCGTGGATGACCAAAC \\
\hline & Antisense: & : CGATTGTGGTGAGTGTGTCTGT & & Antisense: & CGGTTAAAGTGGAATTCTTTCTCG \\
\hline \multirow[t]{2}{*}{ A3 } & Sense: & GGCTATCTGAACTCTATGCATTCG & C6 & Sense: & CACCTTAGGACATAACACACAGACC \\
\hline & Antisense: & : AGGCCATGAGCGTGCGGGTCATA & & Antisense: & CACTTCATCCGGCGGTTCTGGAACC \\
\hline \multirow[t]{2}{*}{ A4 } & Sense: & TGTACCCCTGGATGAAGAAGATCC & $\mathrm{C} 8$ & Sense: & TACCAGACCCTGGAACTGG \\
\hline & Antisense: & : CATTCTCCGGTTCTGAAACCAGATC & & Antisense: & GGAACCAGATTTTGATCTGTCG \\
\hline \multirow[t]{2}{*}{ A5 } & Sense: & AAACTCATTTTGCGGTCGCTAT & $\mathrm{C} 10$ & Sense: & CCACAGGAAATTGGCTGAC \\
\hline & Antisense: & : GTACCTGCCGGAGTGCATGCTC & & Antisense: & CTGCGATTTTGAAACCAGA \\
\hline \multirow[t]{2}{*}{ A6 } & Sense: & GAGTTCCACTTCAACCGCTACC & $\mathrm{C} 11$ & Sense: & GCTGCCCTTATTCGAAATTCCAGAT \\
\hline & Antisense: & : GTACCTGCCGGAGTGCATGCTC & & Antisense: & TGTCGGTCCGTCAGGTTCAGCAT \\
\hline \multirow[t]{2}{*}{ A7 } & Sense: & GAGCTGGAGAAGGAGTTCCA & $\mathrm{C} 12$ & Sense: & TTGCAACTGGCAGAGCTGGAGGGCG \\
\hline & Antisense: & : ACCTTCGTCCTTATGCTCTT & & Antisense: & CTCCGGTTCTGAAACCAGATCTTGA \\
\hline \multirow[t]{2}{*}{ A9 } & Sense: & CTGTTCAACATGTACCTCACCA & $\mathrm{C} 13$ & Sense: & CCCTACACTAAGGTGCAGCTGAA \\
\hline & Antisense: & : CACTCGTCTTTTGCTCGGTC & & Antisense: & TACCTGGCGCTCAGAGAGGTTCGTG \\
\hline \multirow[t]{2}{*}{ A10 } & Sense: & AGAGCAGCAAAGCCTCGCCGGAGAAG & D1 & Sense: & CAATAAGTACTTAACTCGAGCC \\
\hline & Antisense: & : GCTCTCGAGTAAGGTACATATTG & & Antisense: & CGAAGGCTCTTGGGACTGAGAA \\
\hline \multirow[t]{2}{*}{ A11 } & Sense: & GTACTTACTACGTCTCGGGTCCAG & D3 & Sense: & CATCAGCAAGCAGATCTTCC \\
\hline & Antisense: & : AGTCTCTGTGCACGAGCTCCT & & Antisense: & AGCGGTTGAAGTGGAATTCC \\
\hline \multirow[t]{2}{*}{ A13 } & Sense: & ACCTCTGGAAGTCCACTCTGCCC & D4 & Sense: & GGCCTACACCCGGCAGCAAGTCC \\
\hline & Antisense: & : ATGCCTGGCTACCTGGATATGC & & Antisense: & CTTTAGTGTTGGGCAGCTTATGA \\
\hline \multirow[t]{2}{*}{ B2 } & Sense: & TTCGCGGCCGACTCCTGTC & D8 & Sense: & ACAGGTAAAAATCTGGTTCCAG \\
\hline & Antisense: & : CAGGTCGATGGCACAGAGCGTA & & Antisense: & TGTCTTCCTCCAGCTCTTGG \\
\hline \multirow[t]{2}{*}{ B3 } & Sense: & AGGCTGCGGCGCCCCGCAGAAG & D9 & Sense: & TTCGCAGCCGCAGCAGCAGCAACT \\
\hline & Antisense: & : GAAAGGTGGTTGAGGCCATAGA & & Antisense: & TCTCTCTGTTAGGTTGAGAATCCT \\
\hline \multirow[t]{2}{*}{ B4 } & Sense: & GTGCAAAGAGCCCGTCGTCTAC & D10 & Sense: & TGGGTATGAATGTGCATCCT \\
\hline & Antisense: & : CAGAGCGCGTGGGCGATCTCCA & & Antisense: & CTGGAGCATGACAGTGGAGCTGC \\
\hline \multirow[t]{2}{*}{ B5 } & Sense: & GTTCCACTTCAACCGCTACC & D11 & Sense: & ATGAACGACTTTGACGAGTGCGGC \\
\hline & Antisense: & : GCGCTGCCAGCTGTAGCCAGGCTC & & Antisense: & CCACTTCGCGCACGGGCTGGAC \\
\hline \multirow[t]{2}{*}{ B6 } & Sense: & CCTTCTACCGCGAGAAAGAGT & D12 & Sense: & ATGTAGGCGGTGCTGAAATGACCG \\
\hline & Antisense: & : TTGCACGAATTCATCCGCTGCAT & & Antisense: & CCCGCTCTGTAGAGACTGCGCTCA \\
\hline \multirow[t]{2}{*}{ B7 } & Sense: & AGAGTAACTTCCGGATCTA & D13 & Sense: & TCTAAATCAGCCGGACATGTGCGT \\
\hline & Antisense: & : CAGAGCGCGTGCGCGATCTCGATG & & Antisense: & CGGTTCTGAAACCAAATGG \\
\hline
\end{tabular}

B8 Sense: AGCAGCCGCCGGACGCAGGCG Antisense: TCCACTTCATCCTCCGGTTCTG

B9 Sense: CAACTGGCTGCACGCTCGCTCT Antisense: CTCTCACTCAGATTGAGGAGTCT

B13 Sense: TACGCTGATGCCTGCTGTCAATA Antisense: CACAGGCAACAGGGAGTCATGTCG

Primer sequences were obtained from Vider et al (28,30), Alami et al (29) and nucleotide sequence database. NCBI accession numbers of HOXA, HOXB, HOXC and HOXD are NT_007819, NT_086883, NT_086796 and NT_005403, respectively. 
cancer cells show overexpression of HOX genes in ovarian cancer cells. Then, we examined the invasion ability of the cells introduced with an antisense of two overexpressed HOX genes by invasion assay to evaluate whether introduction of the antisense leads to suppression of the invasion ability of cancer cells.

\section{Materials and methods}

Isolation of total RNA from cells and tissues. Five epithelial ovarian cancer cell lines ES-2, SKOV3, CAOV3 (from ATCC), SMOV2 (gifted by Dr K. Yonamine) and JHOC-6 (gifted by Dr H. Ishikawa), were used in this study. These cells were cultured under the supplier's recommendations. At $80 \%$ confluence, the cells were harvested and served in isolation of total RNA. As sources of normal and ovarian cancer tissues, surgical specimens were obtained from women who had undergone gynecological surgery due to benign uterine diseases and ovarian cancer in Asahikawa Medical College Hospital; all had agreed to provide specimens for the study approved by the Ethics Committee of the Asahikawa Medical College. Diagnosis of normal ovarian tissues in all patients was finally confirmed by histopathological examination.

Total RNA from these samples was isolated with RNeasy ${ }^{\mathrm{TM}}$ (Qiagen Inc., USA) after homogenization of the samples. Isolated total RNA $(1 \mu \mathrm{g})$ treated with DNaseI was reversetranscribed into cDNA by Reverse Transcription system ${ }^{\mathrm{TM}}$ (Promega Inc., USA), then subjected to real-time polymerasechain reaction (real-time RT-PCR) using SmartCycler ${ }^{\mathrm{TM}}$ (Cepheid, USA).

Quantitative real-time RT-PCR of ovarian derived samples. Quantitative real-time RT-PCR was applied using gene specific primer sets for the HOX genes (Table I). To adjust the concentrations of reverse-transcribed cDNA from mRNA in each sample, $B$-actin was used as an internal control. Each $25 \mu \mathrm{l}$ of PCR mixture contained $0.3 \mu \mathrm{M}$ of sense and antisense primers, $1 \mu 1$ of cDNA solution, $12.5 \mu 1$ of PCR premixture (Quantiteck SYBR Green PCR Kit ${ }^{\mathrm{TM}}$, Qiagen Inc.) and $6.5 \mu 1$ of supplied water. Typical PCR was performed using first cycle at $95^{\circ} \mathrm{C}$ for $15 \mathrm{~min}$ to activate HotStar Taq polymerase in the premixture, followed by 45 cycles of $94^{\circ} \mathrm{C}$ for $30 \mathrm{sec}, 55^{\circ} \mathrm{C}$ for $30 \mathrm{sec}$, and $72^{\circ} \mathrm{C}$ for $30 \mathrm{sec}$, respectively. Prior to measurement of the gene product by real-time RT-PCR, production of the single target band of each HOX gene was confirmed by electrophoresis using Bioanalyser 2100 (Agilent, USA). Then standard DNA solutions were produced by serial dilutions of columnpurified PCR products (PCR Purification kit, Qiagen Inc.) showing the target band for relative quantitative analysis of HOX and $B$-actin expressions. The original PCR products containing 5-50 ng/ $\mu 1$ of target $\mathrm{HOX}$ and $\beta$-actin fragments were diluted into $10^{-5}, 10^{-7}, 10^{-8}, 10^{-9}$ and $10^{-10}$ with distilled water mixed salmon DNA for establishment of standard curves. The signal intensity in the real-time RT-PCR was measured at $5^{\circ} \mathrm{C}$ lower than melting temperature of each product for each cycle. Relative quantity of each HOX sample was adjusted by ratio of expression of the $\beta$-actin (HOX/ $\beta$-actin) and the degree of expression among each sample was evaluated based on the comparison with the expression of simultaneous-amplified standard solutions. For judgment of overexpression of HOX gene in cancer cells, the average expression level of three normal samples in each $\mathrm{HOX}$ gene was calculated; the expression level of HOX gene in each cancer cell line was then compared with the normal average. Degree of overexpression in the cancer cells is shown numerically or with arrows in cases where the expression in the normal samples was not detected.

Antisense gene introduction of overexpressed HOX genes by electroporation. Sequence of antisense fragments of HOXB7, HOXB13 and HOXC5 as control was designed by sequences from a database (NCBI, Entrez Nucleotide). At first, each antisense fragment was generated by PCR with gene specific primer sets (HOXB7 sense 5'-cattgattatgcgaatgcttt-3', antisense 5'-cgcagtgcatgttgaaggaact-3'; HOXB13 sense 5'-tacgct gatgcctgctgtcaacta-3', antisense 5'-cacaggcaacagggagtcatg tcg-3'; HOXC5 sense 5'-aggtgcaggcatccaggtactg-3', antisense 5'-aggcatccaggtactgctacgg-3'), and then the products were introduced into pGEM-Teasy ${ }^{\mathrm{TM}}$ vector (Invirogen, USA). The sequence of PCR products introduced to the vector was confirmed by DNA sequencer (SEQ4X4 personal sequencing system, Amersham Pharmacia Biotech). The vector was digested with ERI, and fragments were released to be reintroduced into another vector pcDNA3.1 (Invirogen), containing Neo-r gene for selection. The vector $(50 \mu \mathrm{g})$ containing each antisense fragment was lineralized by ApaI and used for electroporation with $2.6 \times 10^{6}$ of SKOV3 cells (Gene Pulser, Bio-Rad). The condition of electroporation was as follows: cuvette gap, $0.4 \mathrm{~cm}$; voltage, $0.25 \mathrm{kV}$; capacitor, $500 \mu \mathrm{F}$. After electroporation, treated cells were cultured for $24 \mathrm{~h}$ prior to use of G418 for selection of the antisenseintroduced cells. Culture with G418 was continued for 5 days; then RT-PCR with isolated total RNA from the cultured cells was carried out to check introduction and expression of the target antisense fragment using primer sets containing sequences of Neo-r and vector itself. Three independent cell lines introduced with each antisense of HOXB7, HOXB13 and HOXC5 were established for the subsequent invasion assay. Simultaneously, mock SKOV3 cell line (introduction of vector sequence only) was also established.

Matrigel assay for the invasion of the cancer cells. To examine the manner of invasion among three cells types, parental SKOV3 cell, mock SKOV3 (no antisense fragment) and antisense-introduced SKOV3, matrigel assay was applied as invasion assay. Cells adjusted to $5 \times 10^{4}$ were put on the membrane of the matrigel chamber (Paragon, USA) and cultured for up to $16 \mathrm{~h}$ after addition of fibronectin as a chemoattractant. After cultivation, the cells moving through matrigel were microscopically counted using $0.1 \%$ trypan blue stain. The ability of invasion of each cell type was calculated as \% invasion and an invasion index was calculated.

\section{Results}

Overexpressed HOX genes in ovarian cancer cells. In this study, we were able to obtain successful amplification for evaluation in 36 out of 39 HOX genes. In HOXB1, HOXC3 
Table II. Overexpression of HOX genes in the ovarian cancer cell lines by real-time RT-PCR.

\begin{tabular}{|c|c|c|c|c|c|c|c|c|c|c|c|c|c|}
\hline Paralog no. & 1 & 2 & 3 & 4 & 5 & 6 & 7 & 8 & 9 & 10 & 11 & 12 & 13 \\
\hline HOXA & & & A3 & A4 & & & A7 & & & A10 & & & A13 \\
\hline SKOV3 & & & & & & & & & & 12.1 & & & $>300$ \\
\hline CAOV3 & & & & & & & 2.1 & & & 13.3 & & & $>300$ \\
\hline JHOC-6 & & & & & & & & & & 3.5 & & & $>300$ \\
\hline SMOV2 & & & 2.0 & & & & & & & & & & $>300$ \\
\hline ES-2 & & & & 2.0 & & & & & & 12.6 & & & $>300$ \\
\hline HOXB & & B2 & B3 & B4 & B5 & B6 & B7 & B8 & B9 & & & & B13 \\
\hline SKOV3 & & 9.2 & 6.6 & 267.0 & 14.9 & 9.4 & $>300$ & 47.6 & 6.1 & & & & $>300$ \\
\hline CAOV3 & & & & 44.9 & & & $>300$ & & 3.1 & & & & $>300$ \\
\hline JHOC-6 & & & & & & & $>300$ & 3.2 & 3.0 & & & & $>300$ \\
\hline SMOV2 & & & & 141.8 & & & $>300$ & & & & & & $>300$ \\
\hline ES-2 & & 4.1 & & 91.8 & & & $>300$ & & 5.6 & & & & $>300$ \\
\hline $\mathrm{HOXC}$ & & & & & & & & & & & & & $\mathrm{C} 13$ \\
\hline CAOV3 & & & & & & & & & & & & & 3.3 \\
\hline HOXD & & & & & & & & & & & & & D13 \\
\hline SKOV3 & & & & & & & & & & & & & 3.6 \\
\hline
\end{tabular}

The numbers indicate folds of expression level of each HOX gene in cancer cells compared with that in normal samples.

a : HOXB7

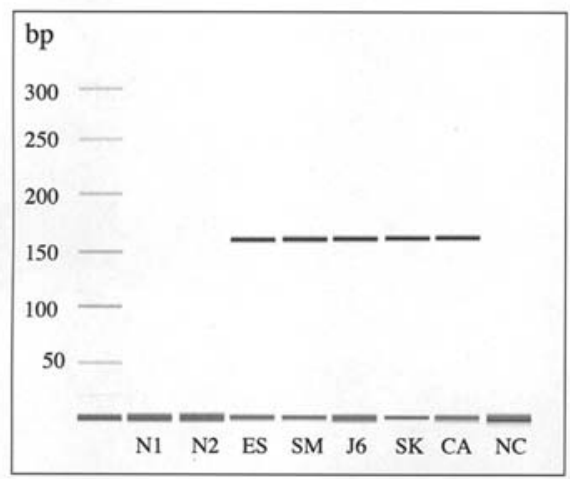

\section{b: HOXB13}

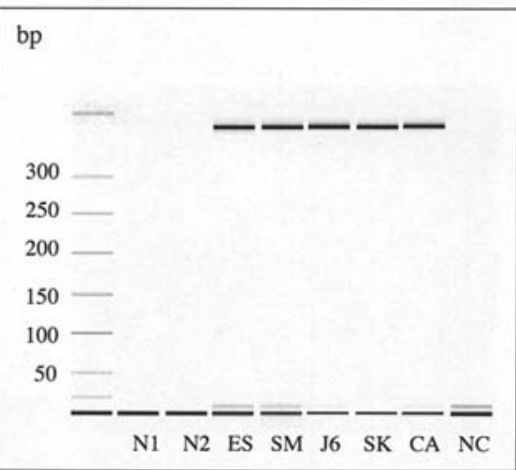

Figure 1. Expressions of HOXB7 and HOXB13 in ovarian cancer cell lines. (a) HOXB7, (b) HOXB13. To visualize bands of all overexpressed HOX genes, RT-PCR was performed at 40 cycles then confirmed by electrophoresis. Overexpression of HOXB7 and HOXB13 was displayed in all cell lines. N1 and N2, normal controls; ES, ES-2; SM, SMOV2; J6, JHOC-6; SK, SKOV3; CA, CAOV3; NC, negative control.

and HOXC9, it is difficult to assess ovarian carcinogenesis because expression of these genes was not observed in either normal or cancer samples, implying the possibility of loss of expression or technical deficiency. In 16 HOX genes, expression in cancer cells was over twice that in normal samples (Table II). HOXA10, HOXA13, HOXB4, HOXB7, HOXB13 and HOXC13 showed little or no expression in normal samples. In the other genes, there was no significant difference of expression level among the three normal controls (data not shown). In HOXA cluster, there are 11 HOX genes, from HOXA1 to HOXA13. Overexpression in cancer cells was seen to various degrees and in various cell lines in HOXA3, HOXA4, HOXA7, HOXA10 and
HOXA13. High levels of overexpression were observed in HOXA10 and HOXA13 in all cells except for HOXA10 of SMOV2. The HOXB cluster consists of 10 genes, overexpression was observed in all $\mathrm{HOXB}$ examined in this study. In HOXB2, HOXB3, HOXB5, HOXB6 and HOXB8, overexpression was revealed in only one or two cell lines, SKOV3 with JHOC-6 or ES-2. However, in HOXB4, HOXB7, HOXB9 and HOXB13, multiple cell lines showed high levels of overexpression (Table II and Fig. 1). In the HOX C and D clusters, only 2 genes, HOXC13 and HOXD13, showed low levels of overexpression that was detected in HOXC13 of CAOV3 and in HOXD13 of SKOV3. Some data suggested loss or reduction of HOX 
a : $\operatorname{HOXB7}$

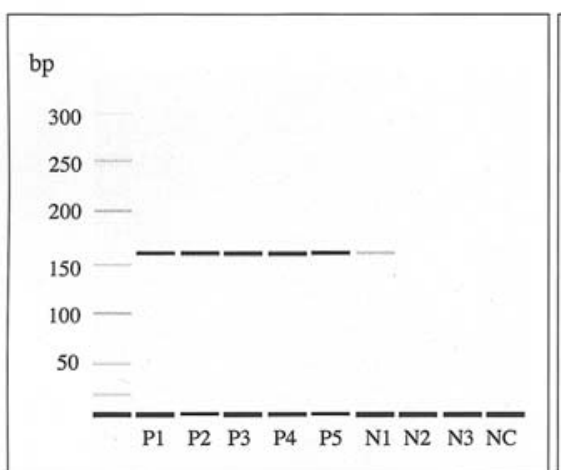

b: HOXB13

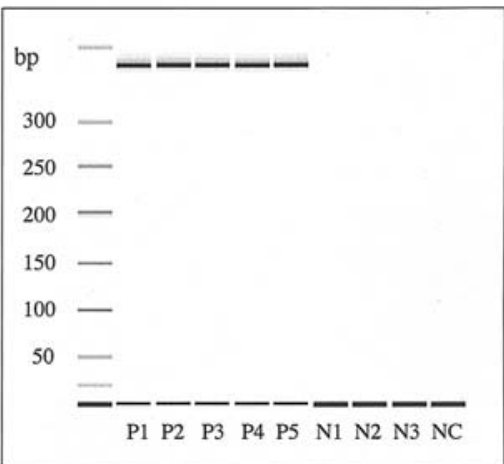

Figure 2. Expressions of HOXB7 and HOXB13 in surgical samples from patients with ovarian cancer. (a) HOXB7, (b) HOXB13. RT-PCR was performed at 48 cycles. Overexpression of HOXB7 and HOXB13 was displayed in all cancer samples and in a normal control with weak expression in HOXB7. No expression was detected in normal controls in HOXB13. P1-P5, samples from different patients with ovarian cancer; N1-N3, normal controls; NC, negative control.

a

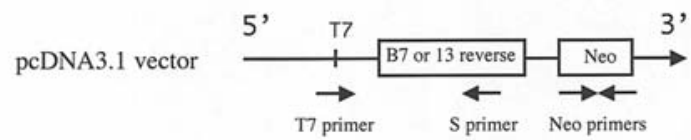

b

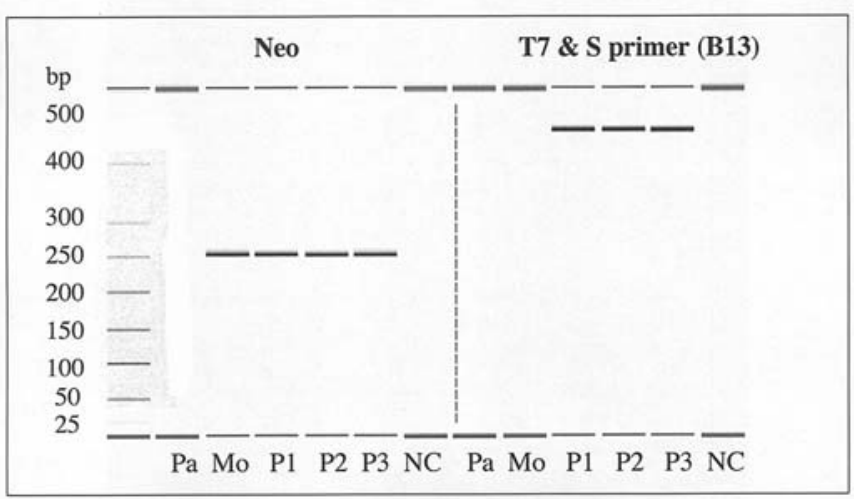

Figure 3. Confirmation of antisense introduction of HOXB13 by RT-PCR. (a) Schematic representation of antisense introduction into pcDNA3.1 vector. Two different types of RT-PCR were performed with a set of T7 and $\mathrm{S}$ primers, and Neo primers, respectively. Internal sequence of HOXB13 was not amplified with the set of T7 and S primer or Neo primers; although the introduced sequence was amplified. In mock cells, the sequence of Neo was only detected because of absence of insert sequence. (b) Expression of introduced sequences in parental cells (SKOV3), mock cells and antisense introduced cells. Pa, parental cells (SKOV3); Mo, mock cells (vector only); P1, P2 and P3, antisense introduced cells; NC, negative control.

expression in cancer cells; however, loss of function was not assessed in this study because pure ovarian surface epithelium from normal controls was not collected.

Chemoinvasion assay of $\mathrm{HOXB7}$ and HOXB13 antisenseintroduced SKOV3 cells. In the HOX genes demonstrating overexpression in this study, fragments of antisense of $\mathrm{HOXB7}$ and HOXB13 that demonstrated high level of overexpression were chosen to investigate behavior of invasiveness of antisense introduced SKOV3 cells. Prior to introduction of antisense fragments, we confirmed overexpression of HOXB7 and HOXB13 in cancer tissues from five different patients with ovarian cancer diagnosed as serous or serous papillary
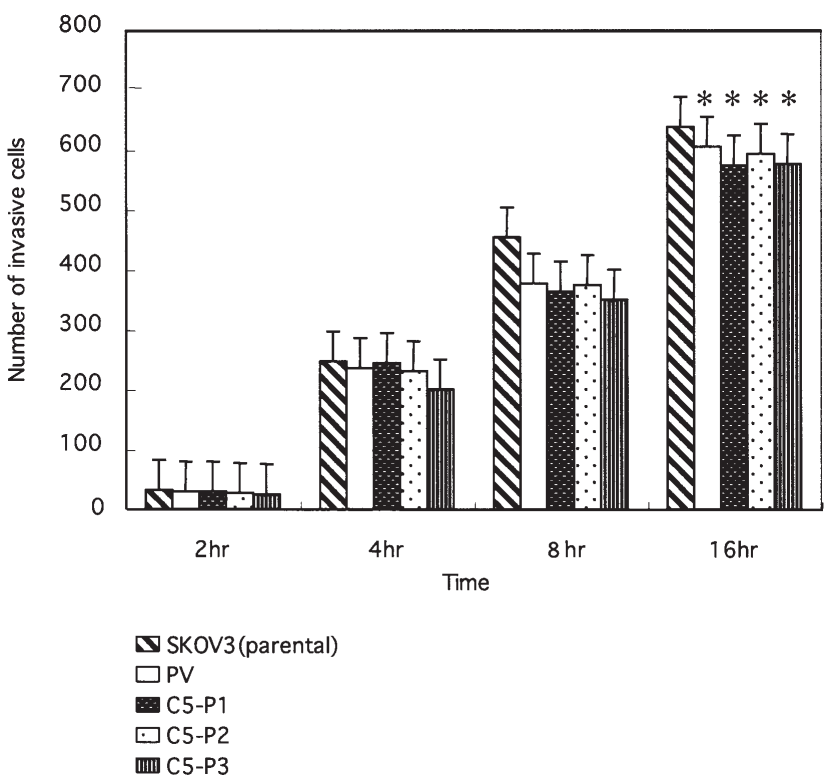

Figure 4. Chemoinvasion assay of antisense HOXC5 introduced cells as a control experiment. Fibronectin was used as chemoattractant. Three independent cell lines, P1, P2 and P3, were established and used for the study. Each cell type was placed on the matrigel chamber and cultured for 2$16 \mathrm{~h}$. The number of invasive cells moving through matrigel is shown. PV, mock cell. No significant difference in cell invasion was detected (indicated by an asterisk).

adenocarcinoma (Fig. 2). The successful introduction of HOXB7 and HOXB13 antisense fragments by electroporation resulted in the establishment of 3 independent cell lines, P1, $\mathrm{P} 2$ and P3 (Fig. 3). In the same way, antisense of HOXC5 that did not show overexpression was also introduced into SKOV3 cells as a normal control. The antisense introduced and mock cells (vector only) served in the matrigel assay with fibronectin as chemoattractants. In HOXB7 and HOXB13, antisense-introduced cells demonstrated suppression of invasiveness in all three lines although parental SKOV3 and mock cells of HOXB7, HOXB13 and all cells introduced antisense. HOXC5 showed expected degree of invasiveness (Figs. 4 and 5). Invasion index indicated nearly 
a

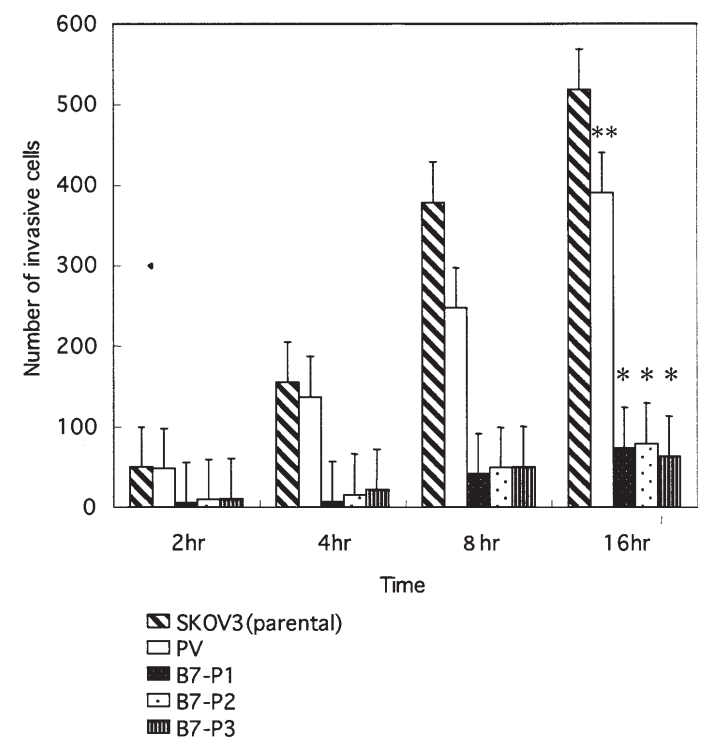

$\mathrm{b}$

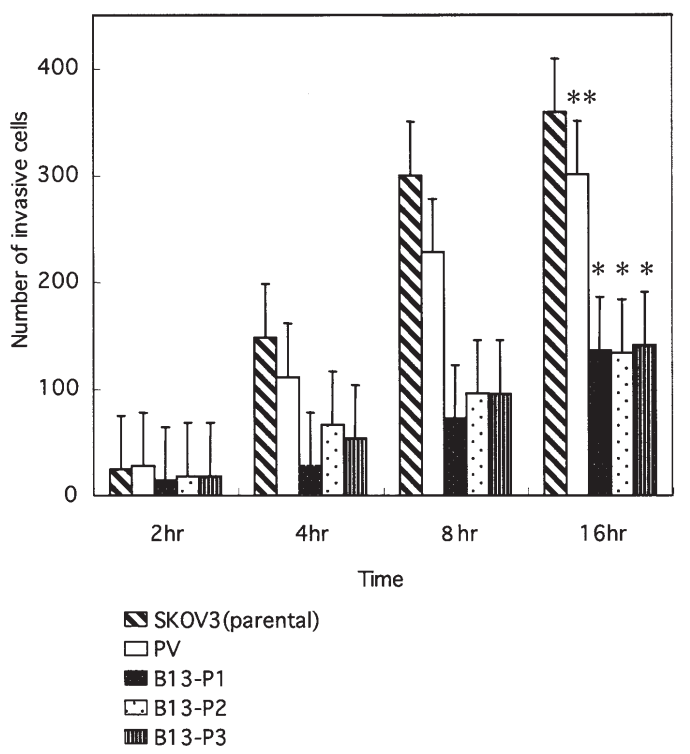

Figure 5. Chemoinvasion assay of overexpressed HOXB7 and HOXB13 antisense introduced cells. (a) HOXB7, (b) HOXB13. No significant difference in cell invasion of PV was detected (indicated by 2 asterisks), however, a significant difference was detected in P1, P2 and P3 of antisense HOXB7 and HOXB13 introduced cells $(\mathrm{P}<0.05$, indicated by an asterisk).

$0.85(85 \%)$ and $0.5(50 \%)$ reduction of invasion ability in HOXB7 and HOXB13 respectively, whereas no difference of invasion index (0.75-0.9) between the parental and mock cells of each HOX gene was shown (Table III).

\section{Discussion}

Recent studies have reported that abnormal HOX expression is involved in a wide variety of human diseases, from metabolic disease to cancer, with regard to different aspects including cell-cycle regulation, cell proliferation, angiogenesis, and invasion or metastasis (31-41). For instance, HOXB4 is involved in stem cell regulation in hematologic cells $(42,43)$, while HOXB7 has a role in angiogenesis (31). HOXA10 is associated with the female reproductive system in the response to sex steroids $(44,45)$ and invasion of breast cancer cells by regulation of p53 (37). Mutation of HOXA13 is the cause of the rare inherited disease, hand-food-genital syndrome (46). HOXB13, has been shown to be involved in differentiation of the epidermal tissue and wound healing $(47,48)$, and has been reported to be important for prostate development (49). These data show the multiple roles of the HOX gene in various stages of human development. Thus, it is highly possible that the role of HOX gene may not be a singular role in ovarian carcinogenesis. We speculated that one of the roles of the HOX gene might be to have the ability of invasion of cancer cells from the viewpoint of regulation of cell movement in embryogenesis.

HOX genes function as a regulatory factor to establish the embryonic body. The female reproductive duct and ovarian surface epithelium are cells formed from the mesodermal layer that are derived from epiblast (ectoderm) through the process of EMT. It is well recognized that fallopian tube, endometrium, cervix and a part of vagina are formed from the Müllerian duct. In contrast, the origin of ovarian surface
Table III. The percent of invasion and invasion index by antisense introduced cells.

\begin{tabular}{|c|c|c|c|c|}
\hline & 2 & 4 & 8 & 16 \\
\hline \multicolumn{5}{|l|}{ HOXB7 } \\
\hline \multicolumn{5}{|l|}{ (\% in:I.I.) } \\
\hline SKOV3 & 49.7 & 155 & 387 & 518 \\
\hline PV & 47.8:0.96 & 137.3:0.89 & 246.9:0.64 & $390.1: 0.75$ \\
\hline PI & 5.5:0.11 & $6.8: 0.04$ & 41.4:0.11 & $73.5: 0.14$ \\
\hline P3 & $9.5: 0.19$ & 15.8:0.10 & 49.1:0.13 & $79.3: 0.15$ \\
\hline P6 & 10.3:0.21 & 21.7:0.14 & 49.8:0.13 & $62.7: 0.12$ \\
\hline \multicolumn{5}{|l|}{ HOXB13 } \\
\hline \multicolumn{5}{|l|}{ (\% in:I.I.) } \\
\hline SKOV3 & 24.5 & 147.9 & 299.8 & 358.8 \\
\hline PV & 27.3:0.87 & 111.1:0.84 & 227.9:0.98 & $300.3: 0.90$ \\
\hline $\mathrm{P} 1$ & $14: 0.66$ & 27.3:0.37 & $71.8: 0.37$ & $135.7: 0.44$ \\
\hline P3 & $18.2: 0.89$ & $66.1: 0.53$ & $95.3: 0.51$ & $135.5: 0.48$ \\
\hline P6 & $18.2: 0.89$ & $53.3: 0.48$ & $95.2: 0.47$ & $140.5: 0.44$ \\
\hline \multicolumn{5}{|c|}{ Mean no. of cells invading through MG insert membrane } \\
\hline \multicolumn{5}{|c|}{$\%$ invasion $=\overline{\text { Mean no. of cells invading through control insert membrane }}$} \\
\hline \multicolumn{5}{|c|}{$\%$ invasion of PV or PI, P3, P6 } \\
\hline \multicolumn{5}{|c|}{ \% invasion of SKOV3 } \\
\hline
\end{tabular}

epithelium is not strictly determined, thus the origin of precursor lesion of ovarian cancer is also undetermined. In a striking paper reported by Cheng et al a possible involvement of histopathological subtypes of ovarian cancer and atypical 
expression of HOX genes was demonstrated (50). However, contribution of HOX genes may not be a single factor in ovarian carcinogenesis like the phenomenon seen in embryogenesis.

This is the first report providing an expression profile of various HOX genes and direct evidence of the overexpressed HOX genes for the invasion ability of ovarian cancer cells. Of 16 HOX genes overexpressed, HOXA10, HOXA13, HOXB4, HOXB7 and HOXB13 showed high levels of overexpression in almost all cancer cells and some in tissues, whereas they were undetectable or had very low expression in normal ovarian tissue, strongly suggesting the potential importance and common roles of those genes in the development of ovarian cancer. In particular, abnormal expression of HOXB7 has been demonstrated to involve immortalization of the cells derived from ovarian tissue by Naora et al (24), indicating substantial roles of HOXB7 in ovarian neoplasm.

To elucidate whether overexpressed HOX genes directly affect the invasion ability of ovarian cancer cells, we introduced an antisense fragment of two highly overexpressed HOX genes, HOXB7 and HOXB13. The HOXB7 was selected because it has been reported to contribute to ovarian neoplasms (24), while we have also found HOXB13 overexpression in endometrial cancer (data not shown) (27). A marked reduction of invasion ability was seen in HOXB7 (85\%) and HOXB13 (50\%). By contrast, mock cells introduced to an antisense of HOXB7 or HOXB13, and cells introduced to HOXC5 did not show obvious difference in invasion ability. Taken together, overexpressed HOX genes are directly involved in the invasion ability of the ovarian cancer cells. However, in neither experiment did the invasion index drop to $0 \%$ indicating complete suppression. These results suggest that the function of the HOX genes for invasion may be redundant and complemented by other paralogous genes or the genes in the same cluster as the function seen in the embryogenesis. In fact, overexpression of the HOXA7 and HOXA13 that are paralogous genes of HOXB7 and HOXB13, were also observed in this experiment.

It is not clear yet how overexpression of these HOX genes contributes to invasive characteristics of ovarian cancer cells. Investigation to find correlation between abnormal HOX expression and the function of other related genes that mediate cell adhesion such as MMPs, ETS, uPA, PAI, B-catenin, Ecadherin and Snail, are now under way. However, it is possible to associate HOXB7 and HOXB13 with neovascularization of the cancer tissue that is important to invasion. HOXB13 may function as a gene for wound healing of the damaged portions of the ovarian and endometrial epithelium due to monthly ovulation and menstruation, while HOXB7 supports angiogenesis of the ovarian neoplasms.

\section{Acknowledgments}

We thank Dr H. Ishikawa and Dr K. Yonamine for providing cancer cells. We thank Miss K. Kirio for collecting samples. This study was supported by a Grant-in-Aid for General Scientific Research No. 13671687 and 17591710 from the Ministry of Education, Science, Sports and Culture, Japan.

\section{References}

1. Berek JS, Adashi EY and Hillard PA (eds): Novak's Gynecology. 12th edition. Williams \& Wilkins, Baltimore, pp1155-1230, 1996.

2. Hay ED: An overview of epithelio-mesenchymal transformation. Acta Anat 154: 8-20, 1995.

3. Hay ED and Zuk A: Transformations between epithelium and mesenchyme: normal, pathological, and experimentally induced. Am J Kidney Dis 26: 678-690, 1995.

4. Ellenrieder V, Buck A and Gress TM: TGF beta-regulated transcriptional mechanisms in cancer. Int J Gastrointest Cancer 31: 61-69, 2002.

5. Yi JY, Hur KC, Lee E, Jin YJ, Arteaga CL and Son YS: TGF beta1-mediated epithelial to mesenchymal transition is accompanied by invasion in the SiHa cell line. Eur J Cell Biol 81: 457-468, 2002.

6. Janda E, Lehmann K, Killisch I, et al: Ras and TGF[beta] cooperatively regulate epithelial cell plasticity and metastasis: dissection of Ras signaling pathways. J Cell Biol 156: 299-313, 2002.

7. Oft M, Akhurst RJ and Balmain A: Metastasis is driven by sequential elevation of H-ras and Smad2 levels. Nat Cell Biol 4: 487-494, 2002.

8. Yokoyama K, Kamata N, Fujimoto R, et al: Increased invasion and matrix metalloproteinase-2 expression by Snail-induced mesenchymal transition in squamous cell carcinomas. Int $\mathbf{J}$ Oncol 22: 891-898, 2003

9. Blanco MJ, Moreno-Bueno G, Sarrio D, Locascio A, Cano A, Palacios J and Nieto MA: Correlation of Snail expression with histological grade and lymph node status in breast carcinomas. Oncogene 21: 3241-3246, 2002.

10. Samuel S and Bernstein LR: Adhesion, migration, transcriptional, interferon-inducible, and other signaling molecules newly implicated in cancer susceptibility and resistance of JB6 cells by cDNA microarray analyses. Mol Carcinog 39: 34-60, 2004.

11. McGinnis W and Krumlauf R: Homeobox genes and axial patterning. Cell 68: 283-302, 1992.

12. Mark M, Rijli FM and Chambon P: Homeobox genes in embryogenesis and pathogenesis. Pediatr Res 42: 421-429, 1997.

13. Maroulakou IG and Spyropoulos DD: The study of HOX gene function in hematopoietic, breast and lung carcinogenesis. Anticancer Res 23: 2101-2110, 2003.

14. Lawrence HJ, Sauvageau G, Humphries RK and Largman C: The role of HOX homeobox genes in normal and leukemic hematopoiesis. Stem Cells 14: 281-291, 1996.

15. Buske C and Humphries RK: Homeobox genes in leukemogenesis. Int J Hematol 71: 301-308, 2000.

16. Raman V, Martensen SA, Reisman D, et al: Compromised HOXA5 function can limit p53 expression in human breast tumours. Nature 405: 974-978, 2000.

17. Cantile M, Pettinato G, Procino A, Feliciello I, Cindolo L and Cillo C: In vivo expression of the whole HOX gene network in human breast cancer. Eur J Cancer 39: 257-264, 2003.

18. Cantile M, Cindolo L, Napodano G, Altieri V and Cillo C: Hyperexpression of locus $\mathrm{C}$ genes in the HOX network is strongly associated in vivo with human bladder transitional cell carcinomas. Oncogene 22: 6462-6468, 2003.

19. Miller GJ, Miller HL, van Bokhoven A, et al: Aberrant HOXC expression accompanies the malignant phenotype in human prostate. Cancer Res 63: 5879-5888, 2003.

20. Waltregny D, Alami Y, Clausse N, De Leval J and Castronovo V: Overexpression of the homeobox gene HOXC8 in human prostate cancer correlates with loss of tumor differentiation. Prostate 50: 162-169, 2002.

21. Calvo R, West J, Franklin W, et al: Altered HOX and WNT7A expression in human lung cancer. Proc Natl Acad Sci USA 97: 12776-12781, 2000.

22. Takahashi Y, Hamada J, Murakawa K, et al: Expression profiles of 39 HOX genes in normal human adult organs and anaplastic thyroid cancer cell lines by quantitative real-time RT-PCR system. Exp Cell Res 293: 144-153, 2004.

23. Hung YC, Ueda M, Terai Y, et al: Homeobox gene expression and mutation in cervical carcinoma cells. Cancer Sci 94: 437-441, 2003.

24. Naora H, Yang YQ, Montz FJ, Seidman JD, Kurman RJ and Roden RB: A serologically identified tumor antigen encoded by a homeobox gene promotes growth of ovarian epithelial cells. Proc Natl Acad Sci USA 98: 4060-4065, 2001. 
25. Naora H, Montz FJ, Chai CY and Roden RB: Aberrant expression of homeobox gene HOXA7 is associated with mullerian-like differentiation of epithelial ovarian tumors and the generation of a specific autologous antibody response. Proc Natl Acad Sci USA 98: 15209-15214, 2001.

26. Osborne J, Hu C, Hawley C, Underwood LJ, O'Brien TJ and Baker VV: Expression of HOXD10 gene in normal endometrium and endometrial adenocarcinoma. J Soc Gynecol Investig 5: 277-280, 1998.

27. Zhao Y, Yamashita T and Ishikawa M: Regulation of tumor invasion by HOXB13 gene that is overexpressed in human endometrial cancer AN3CA cells. Oncol Rep 13: 721-726, 2005.

28. Vider BZ, Zimber A, Hirsch D, et al: Human colorectal carcinogenesis is associated with deregulation of homeobox gene expression. Biochem Biophys Res Commun 232: 742-748, 1997.

29. Alami Y, Castronovo V, Belotti D, Flagiello D and Clausse N: HOXC5 and HOXC8 expression are selectively turned on in human cervical cancer cells compared to normal keratinocytes. Biochem Biophys Res Commun 257: 738-745, 1999.

30. Vider BZ, Zimber A, Chastre E, et al: Deregulated expression of homeobox-containing genes, $\mathrm{HOXB} 6, \mathrm{~B} 8, \mathrm{C} 8, \mathrm{C} 9$, and $\mathrm{Cdx}-1$, in human colon cancer cell lines. Biochem Biophys Res Commun 272: 513-518, 2000.

31. Care A, Felicetti F, Meccia E, et al: HOXB7: a key factor for tumor-associated angiogenic switch. Cancer Res 61: 6532-6539, 2001.

32. Boudreau N, Andrews C, Srebrow A, Ravanpay A and Cheresh DA: Induction of the angiogenic phenotype by Hox D3. J Cell Biol 139: 257-264, 1997.

33. Myers C, Charboneau A and Boudreau N: Homeobox B3 promotes capillary morphogenesis and angiogenesis. J Cell Biol 148: 343-351, 2000.

34. Uyeno LA, Newman-Keagle JA, Cheung I, Hunt TK, Young DM and Boudreau N: Hox D3 expression in normal and impaired wound healing. J Surg Res 100: 46-56, 2001.

35. Boudreau NJ and Varner JA: The homeobox transcription factor Hox D3 promotes integrin alpha5beta1 expression and function during angiogenesis. J Biol Chem 279: 4862-4868, 2004.

36. Cillo C: HOX genes in human cancers. Invasion Metastasis 14: 38-49, 1994.

37. Chu MC, Selam FB and Taylor HS: HOXA10 regulates p53 expression and matrigel invasion in human breast cancer cells. Cancer Biol Ther 3: 568-572, 2004.
38. Redline RW, Hudock P, MacFee M and Patterson P: Expression of AbdB-type homeobox genes in human tumors. Lab Invest 71: 663-670, 1994.

39. Fisher D and Mechali M: Vertebrate HoxB gene expression requires DNA replication. EMBO J 22: 3737-3748, 2003.

40. Gabellini D, Colaluca IN, Vodermaier HC, et al: Early mitotic degradation of the homeoprotein HOXC10 is potentially linked to cell cycle progression. EMBO J 22: 3715-3724, 2003.

41. Wu Y, Moser M, Bautch VL and Patterson C: HoxB5 is an upstream transcriptional switch for differentiation of the vascular endothelium from precursor cells. Mol Cell Biol 23: 5680-5691, 2003

42. Amsellem S, Pflumio F, Bardinet D, et al: Ex vivo expansion of human hematopoietic stem cells by direct delivery of the HOXB4 homeoprotein. Nat Med 9: 1423-1427, 2003.

43. Antonchuk J, Sauvageau G and Humphries RK: HOXB4 overexpression mediates very rapid stem cell regeneration and competitive hematopoietic repopulation. Exp Hematol 29: 1125-1134, 2001.

44. Cermik D, Karaca M and Taylor HS: HOXA10 expression is repressed by progesterone in the myometrium: differential tissue-specific regulation of HOX gene expression in the reproductive tract. J Clin Endocrinol Metab 86: 3387-3392, 2001.

45. Taylor HS, Arici A, Olive D and Igarashi P: HOXA10 is expressed in response to sex steroids at the time of implantation in the human endometrium. J Clin Invest 101: 1379-1384, 1998.

46. Goodman FR, Bacchelli C, Brady AF, et al: Novel HOXA13 mutations and the phenotypic spectrum of hand-foot-genital syndrome. Am J Hum Genet 67: 197-202, 2000.

47. Judith A, Mack LL, Nobuyuki S, Vincent CH and Edward VM: Hoxb13 up-regulates transglutaminase activity and drives terminal differentiation in an epidermal organotypic model. J Biol Chem 280: 29904-29911, 2005

48. Mack JA, Abramson SR, Ben Y, et al: Hoxb13 knockout adult skin exhibits high levels of hyaluronan and enhanced wound healing. FASEB J 17: 1352-1354, 2003.

49. Economides KD and Capecchi MR: Hoxb13 is required for normal differentiation and secretory function of the ventral prostate. Development 130: 2061-2069, 2003

50. Cheng W, Liu J, Yoshida H, Rosen D and Naora H: Lineage infidelity of epithelial ovarian cancers is controlled by HOX genes that specify regional identity in the reproductive tract. Nat Med 11: 531-537, 2005. 